\title{
FRECUENCIA Y FACTORES DE RIESGO PARA BACTERIEMIA POR ENTEROBACTERIAS PRODUCTORAS DE BETALACTAMASA DE ESPECTRO EXTENDIDO EN PACIENTES DE UN HOSPITAL PÚBLICO DE LIMA, PERÚ
}

\author{
Angelo Falconí Sarmiento ${ }^{1, a}$, Monthy Nolasco Mejia1,b, Alonzo Bedoya Rozas ${ }^{1, c}$, Catherine Amaro Giraldo ${ }^{1,2, d}$ \\ Germán Málaga ${ }^{1,2, e}$
}

\begin{abstract}
RESUMEN
Con el objetivo de determinar la frecuencia y factores de riesgo para bacteriemia por enterobacterias productoras de betalactamasa de espectro extendido (BLEE) en pacientes internados en un hospital público de Lima se realizó un estudio transversal. Fueron incluidos pacientes mayores de 14 años, con hemocultivos positivos durante su hospitalización en el Hospital Nacional Cayetano Heredia el 2016. Se clasificó a los pacientes según la bacteria aislada (productora o no de BLEE). El 50,6 \% de las bacteriemias fueron causadas por enterobacterias productoras de BLEE, 55,8 \% y 32,6 \% por E. Coli y K. pneumoniae, respectivamente. No hallándose diferencias con relación a comorbilidades, ni uso previo de antibióticos $(62,8 \%$ de las bacteriemias por cepas productoras de BLEE y en $57 \%$ en las no productoras $(p=0,595))$. La mitad de las bacteriemias por enterobacterias en pacientes hospitalizados son producidas por enterobacterias productoras de BLEE, y de estas, el $40 \%$ son adquiridas en la comunidad.
\end{abstract}

Palabras Clave: Enterobacterias; Bacteriemia; Beta-Lactamasas; Factores de riesgo. (fuente: DeCS BIREME).

\section{FREQUENCY AND RISK FACTORS FOR BACTEREMIA CAUSED BY EXTENDED- SPECTRUM BETA-LACTAMASE-PRODUCING ENTEROBACTERIACEAE IN PATIENTS OF A PUBLIC HOSPITAL IN LIMA, PERU}

\begin{abstract}
A cross-sectional study was conducted aimed at determining the frequency and the risk factors for bacteremia caused by extended-spectrum Beta-Lactamase (ESBL)-producing Enterobacteriaceae in patients hospitalized in a public hospital in Lima. The study included patients over 14 years of age, with positive blood cultures during their hospitalization in Hospital Nacional Cayetano Heredia in 2016. Patients were classified according to the isolated bacterium (ESBL-producing or not). Bacteremia was caused by ESBL-producing Enterobacteriacea in $50.6 \%$ of the cases; $55.8 \%$ and $32.6 \%$ by $E$. Coli and $K$. pneumoniae, respectively. No differences were found regarding co-morbidity, or prior antibiotic use $(62.8 \%$ of bacteremia due to ESBLproducing strains and $57 \%$ in the non-producing strains $[\mathrm{p}=0.595]$ ). Half of the bacteremia cases due to Enterobacteriaceae in hospitalized patients are produced by ESBL-producing Enterobacteriaceae and, of these, $40 \%$ are acquired in the community.
\end{abstract}

Keywords: Enterobacteriaceae; Bacteremia; Beta-Lactamases; Risk factors. (source: MeSH NLM).

\section{INTRODUCCIÓN}

Estamos asistiendo a un incremento en los casos de bacteriemias en pacientes hospitalizados y de la mortalidad relacionada ${ }^{(1)}$, así en Europa se incrementó en 71 \% las cepas de E. coli productora de ß-lactamasas de espectro extendido (BLEE) entre el 2002 y 2009 (2); en China, entre el 2010 y 2012, la proporción incrementó de 19,8 \% a 23 \% (3); en Brasil, se observó que la mortalidad por infección por $K$. pneumoniae productora de BLEE es alta y más del $50 \%$ de los pacientes fallecidos se debió a un inadecuado tratamiento antibiótico inicial ${ }^{(4)}$. En Colombia, fueron descritos como factores de riesgo para estas infecciones el sexo femenino, enfermedad cardiovascular y el uso previo de antibióticos como ceftriaxona y carbapenémicos ${ }^{(5)}$. En el Perú un estudio retrospectivo evidenció una mortalidad atribuible a bacteriemias por enterobacterias de $41,2 \%$ de las cuales $54,3 \%$ pertenecieron al grupo productor de BLEE (1).

Las enterobacterias BLEE son capaces de hidrolizar a las oximino-cefalosporinas y monobactámicos y suelen acompañarse de mecanismos de resistencia contra

\footnotetext{
Universidad Peruana Cayetano Heredia. Lima, Perú.

Hospital Cayetano Heredia. Lima, Perú.

Médico cirujano; ${ }^{\mathrm{b}}$ médico cirujano; ${ }^{\mathrm{c}}$ médico cirujano; ${ }^{\mathrm{d}}$ médico patóloga clínica, egresada de la maestria de microbiologia; ${ }^{\mathrm{e}}$ medico internista, master en epidemiologia

Recibido: 17/06/2017 Aprobado: 28/02/2018 En línea: 05/04/2018
}

Citar como: Falconí-Sarmiento A, Nolasco-Mejia M, Bedoya-Rozas A, Amaro-Giraldo C, Málaga G. Frecuencia y factores de riesgo para bacteriemia por enterobacterias productoras de BLEE en pacientes de un hospital público de Lima, Perú. Rev Peru Med Exp Salud Publica. 2018;35(1):62-7. doi: 10.17843/rpmesp.2018.351.3601. 
fluoroquinolonas, aminoglucósidos, trimetoprim-sulfametoxazol entre otros, lo cual dificulta su tratamiento ${ }^{(6)}$. El tratamiento antibiótico recomendado son los carbapenems ${ }^{(7)}$.

A nivel mundial, las enterobacterias son la familia de bacterias más frecuentemente asociadas a infecciones intrahospitalarias, siendo Escherichia coli y Klebsiella spp., las vinculadas a producción de BLEE. En el caso de la $E$. coli, la resistencia asociada a la producción de BLEE es atribuida a la transmisión de plásmidos entre las cepas, mientras que en el caso de $K$. pneumoniae, la diseminación de cepas clonales es más frecuente ${ }^{\left({ }^{(8)}\right.}$. En hospitales de Perú se han aislado en sangre cepas de E. coli con alta resistencia a cefalosporinas y quinolonas (9). Se han reportado múltiples tipos de beta lactamasas codificadas según los distintos tipos de genotipo encontrados en las diversas regiones alrededor del mundo ${ }^{(10)}$.

Algunos de los factores de riesgo descritos en la literatura son la edad, comorbilidades, infecciones de tracto urinario recurrentes, estancia hospitalaria prolongada, estancia en la unidad de cuidados intensivos ( $\mathrm{UCl}$ ), uso previo de antibióticos y colonización por gérmenes productores de BLEE (11). Asimismo, ha sido reportado como factor de riesgo importante para el desarrollo de bacteriemia, al uso de catéteres, especialmente catéter venoso central (CVC), siendo su incidencia determinada por el tipo de catéter, frecuencia de manipulación y severidad de la enfermedad previa ${ }^{(5,12)}$. Este es un problema en aumento y que genera una mayor demanda económica ${ }^{(13)}$. En pacientes sometidos a hemodiálisis, las infecciones de catéter y otras complicaciones del acceso vascular son la mayor causa de morbilidad y mortalidad; siendo Klebsiella spp. causante de creciente mortalidad, afectando sobre todo a adultos mayores, malnutridos, con estancia prolongada (mayor a 30 días), portadores de comorbilidades y evolución tórpida (shock, intubación, estancia en UCl) ${ }^{(11,14)}$.

Por tal motivo, el objetivo del presente estudio es describir la frecuencia y factores de riesgo para bacteriemia por enterobacterias productoras de BLEE en pacientes internados en un hospital público de Lima, Perú.

\section{EL ESTUDIO}

\section{DISEÑO Y POBLACIÓN}

Estudio descriptivo de corte transversal, que incluyó a todos los pacientes mayores de 14 años, en los que se hubiera aislado al menos un hemocultivo con presencia de alguna enterobacteria durante su hospitalización en el Hospital Nacional Cayetano Heredia entre enero a diciembre del 2016 y clasificados según el tipo de bacteriemia en: bacteria productora de BLEE o no, según criterios estándares internacionales del Clinical and Laboratory Standards Institute (CLSI) ${ }^{(15)}$, se revisaron además las historias

\section{MENSAJES CLAVE}

Motivación para realizar el estudio. En los últimos años se ha visto un incremento en la incidencia y mortalidad de bacteriemia por enterobacterias productoras de betalactamasa de espectro extendido.

Principales hallazgos. La mitad de bacteriemias producidas por enterobacterias, en pacientes de un hospital público de Lima, serían producidas por gérmenes productores de betalactamasa de espectro extendido, siendo E. coli y K. pneumoniae las especies más frecuentes y de estas, el $40 \%$ son adquiridas en la comunidad.

Implicancias. La alta frecuencia de infecciones por patógenos productores de betalactamasa de espectro extendido en la comunidad y en pacientes hospitalizados es recurrente, por lo que se deberían tomar cultivos de manera imperativa en todos los casos, ante la posibilidad de estar frente a la presencia de gérmenes productores de betalactamasa de espectro extendido, de manera que se pueda dirigir la terapia antibiótica de manera óptima.

clínicas de donde se obtuvo información de caracteristicas sociodemográficas y clínicas.

\section{DEFINICIONES}

Bacteriemia por enterobacterias: detección en uno o más hemocultivos positivos para alguna enterobacteria (Escherichia coli, Klebsiella pneumoniae, Enterobacter spp, Salmonella spp, Citrobacter freunii, Serratia spp y Proteus mirabilis) ${ }^{(8)}$.

Productoras de BLEE: bacterias en las que el antibiograma mostraran resistencia o susceptibilidad intermedia, en la prueba de difusión en disco o concentración mínima inhibitoria, a cefalosporinas de primera, segunda y tercera generación y aztreonam ${ }^{(16)}$.

Detección microbiana: Sistema VersaTREK®, que detecta microorganismos por medio de variación de presiones por consumo o producción de gases. Las muestras positivas fueron procesadas en el sistema automatizado VITEK 2, que permite identificación bacteriana mediante la inoculación de una suspensión de microorganismos en tarjetas con determinados paneles de reacciones bioquímicas y establece la sensibilidad antimicrobiana mediante el sistema de MIC el cual se lleva a cabo a través de tarjetas que contienen diluciones estandarizadas de distintos antibióticos correspondientes a los puntos de corte de sensibilidad establecidos por el CLSI (15,17). Si las muestras no podían ser procesadas en el sistema VITEK, se hacía manualmente, procediendo a la identificación de las bacterias mediante el sembrado en los medios MacConkey, Agar Chocolate y Agar Sangre, luego proceder a la realización del antibiograma mediante la prueba de difusión de disco y finalmente, el laboratorio reportaba el género y especie de la bacteria encontrada, el antibiograma correspondiente a los resultados obtenidos de manera 
manual o automática y en las observaciones reportaba si las cepas eran productoras de BLEE.

Adquisición nosocomial: bacteriemia producida después de transcurridas 48 horas de hospitalización, o aparición de la bacteriemia antes de los 30 días posteriores a una estancia hospitalaria. Dentro de esta categoría se incluyó a aquellos pacientes que frecuentan el ambiente hospitalario (quimioterapia ambulatoria, terapia dialítica, rehabilitación).

\section{ANÁLISIS ESTADÍSTICO}

Se resumen los datos en tablas usando porcentajes, mediana e intervalos intercuartiles. Posteriormente, se realizó análisis bivariado por prueba de $U$ de Mann Whitney para variables numéricas y para las variables categóricas se realizó el test de Chi-cuadrado y exacta de Fischer, para determinar la asociación entre la infección por enterobacterias productoras de BLEE y las otras variables. Valores de $p$ inferiores a 0.05 se consideran significativos. Se utilizó el programa estadístico STATA 14.

\section{CONSIDERACIONES ÉTICAS}

El presente estudio fue evaluado y aprobado por el comité de ética del Hospital Cayetano Heredia, y por el de la Universidad Peruana Cayetano Heredia.

\section{RESULTADOS}

Hubo 105 casos de bacteriemias por enterobacterias. De estos, siete fueron casos repetidos y 13 historias no se hallaron en archivo; por lo que la muestra estudiada estuvo constituida por 85 casos.

La mediana de edad de la población estudiada fue de 62 años (rango intercuartil: 44-74) y 45 (52,9\%) fueron de sexo femenino. La mayor frecuencia de infecciones fue por E. coli (50,5\%), seguida por K. pneumoniae (29,4\%). La mayor parte de las infecciones sistémicas provino de origen urinario e intraabdominal representando el $49,4 \%$ y $16,4 \%$ respectivamente. Once pacientes (12,9\%) requirieron internamiento en $\mathrm{UCI}$ y diez $(11,7 \%)$ pacientes fallecieron durante la hospitalización (Tabla 1).

El 50,5\% de las infecciones encontradas, fueron por cepas productoras de BLEE. En la comparación realizada sobre las características de las poblaciones, según su condición o no de producción de BLEE, no hallamos diferencias en cuanto a demografía (edad, estancia hospitalaria), ni en factores de riesgo descritos (anemia, desnutrición, uso previo de antibióticos), ni en cuanto a la distribución general (E. coli seguida por K. pneumoniae 55,8 \% y 32,5\% respectivamente), ni en lo referente al punto de partida de la infección (Tabla 1).
La mortalidad en la población con infección por enterobacterias productoras de BLEE fue $16,2 \%$, mientras que en los pacientes con infección producida por enterobacteria no productora de BLEE fue $7,1 \%$; esa diferencia no fue estadísticamente significativa $(p=0,313)$.

\section{DISCUSIÓN}

La mitad de las bacteriemias producidas por enterobacterias, son cepas productoras de BLEE, resultado preocupante no solo porque estaríamos constatando un incremento de frecuencia de infecciones por enterobacterias productoras de BLEE (comparado con el $34,6 \%$ en 2009); sino que sería la más alta reportada en Sudamérica (16). Esta elevada frecuencia de enterobacterias resistentes productoras de BLEE, podría tener explicación en el pobre cumplimiento a la terapia antibiótica prescrita o a la elevada frecuencia de automedicación de hasta $75 \%{ }^{(18)}$.

Otro hallazgo importante es que hasta el $40 \%$ de las infecciones producidas por cepas productoras de BLEE fueron adquiridas en la comunidad y que el foco de origen más frecuente de estas cepas fue el urinario, hallazgo que ratifica lo encontrado previamente en relación de que hasta el $40 \%$ de las infecciones urinarias provenientes de la comunidad son causadas por cepas productoras de BLEE ${ }^{(19)}$. Nuestra frecuencia de bacteriemias producidas por enterobacterias productoras de BLEE adquiridas en la comunidad, también sería la más alta reportada comparado con $23 \%$ y $35 \%$ en Europa y Latinoamérica, respectivamente ${ }^{(6,10)}$.

Hicimos la búsqueda exploratoria de factores asociados, como uso previo de antibióticos, variables como edad, sexo, así como el uso de dispositivos invasivos reportadas en otros estudios como significativas ${ }^{(20)}$. Sin embargo, no se encontraron asociaciones significativas, tampoco hallamos mayor mortalidad comparado con otros estudios ${ }^{(1,10)}$, tal vez por no haber tenido suficiente poder para encontrar diferencias.

En cuanto a la mortalidad, si bien no detectamos diferencias según la condición de infección por patógenos productores o no de BLEE, esta ocurrió en los primeros siete días, cuando lo descrito es que suele incrementarse a partir del día 12 de hospitalización ${ }^{(1,18)}$, este hallazgo, podría ser un elemento interesante a explorar y determinar si esta temprana mortalidad en la hospitalización, es consecuencia de la virulencia del germen implicado, retraso en la búsqueda de ayuda médica, las insuficientes o inadecuadas medidas de resucitación o en el inicio tardío de antibióticos, ${ }^{(18,20)}$ o por el contrario, como consecuencia de una cobertura antibiótica empírica inapropiada.

A diferencia de nuestro estudio donde el porcentaje de pacientes con bacteriemias por enterobacterias productoras de BLEE con ingreso a la unidad de cuidados intensivos 
Tabla 1. Características sociodemográficas, de la infección, hospitalarias, antecedentes y complicaciones en pacientes hospitalizados con bacteriemia por enterobacterias productoras de ß-lactamasas de espectro extendido (BLEE)

\begin{tabular}{|c|c|c|c|c|c|c|c|}
\hline \multirow[b]{2}{*}{ Variables } & \multicolumn{2}{|c|}{ Total } & \multicolumn{2}{|c|}{ BLEE (+) } & \multicolumn{2}{|c|}{ BLEE (-) } & \multirow[b]{2}{*}{ Valor de $p$} \\
\hline & $n=85$ & $\%$ & $n=43$ & $\%$ & $n=42$ & $\%$ & \\
\hline \multicolumn{8}{|l|}{ Sociodemográficas } \\
\hline Edad $^{*}$ & 62 & $44-74$ & 63 & $42-75$ & 62 & $46-72$ & 0,847 \\
\hline \multicolumn{8}{|l|}{ Sexo } \\
\hline Masculino & 40 & 47,1 & 19 & 44,2 & 21 & 50,0 & 0,591 \\
\hline Femenino & 45 & 52,9 & 24 & 55,8 & 21 & 50,0 & \\
\hline \multicolumn{8}{|l|}{ Infección } \\
\hline \multicolumn{8}{|l|}{ Patógeno aislado } \\
\hline E. coli & 43 & 50,6 & 24 & 55,8 & 19 & 45,2 & 0,149 \\
\hline Klebsiella pneumoniae & 25 & 29,4 & 14 & 32,6 & 11 & 26,2 & \\
\hline Otras $t$ & 17 & 20,0 & 5 & 11,6 & 12 & 28,6 & \\
\hline \multicolumn{8}{|l|}{ Origen $^{* *}$} \\
\hline Urinario & 42 & 49,4 & 24 & 55,8 & 18 & 42,9 & 0,755 \\
\hline Intra-abdominal & 14 & 16,5 & 6 & 14,0 & 8 & 19,0 & \\
\hline Catéter & 10 & 11,8 & 5 & 11,6 & 5 & 11,9 & \\
\hline Pulmonar & 8 & 9,4 & 4 & 9,3 & 4 & 9,5 & \\
\hline Otros & 11 & 12,9 & 4 & 9,3 & 7 & 16,7 & \\
\hline Infección Nosocomial & 53 & 62,4 & 25 & 58,1 & 28 & 66,7 & 0,417 \\
\hline \multicolumn{8}{|l|}{ Hospitalarias } \\
\hline Uso de dispositivos invasivos $\neq$ & 25 & 47,2 & 15 & 60,0 & 10 & 35,7 & 0,077 \\
\hline Días de estancia hospitalaria * & 15 & $10-21$ & 16 & $11-22$ & 12 & $7-18$ & 0,086 \\
\hline Internamiento en $\mathrm{UCl}$ & 11 & 12,9 & 6 & 14,0 & 5 & 11,9 & 0,778 \\
\hline \multicolumn{8}{|l|}{ Complicaciones } \\
\hline Disfunción renal & 36 & 42,4 & 20 & 46,5 & 16 & 38,1 & 0,432 \\
\hline \multicolumn{8}{|l|}{ Antecedentes } \\
\hline Anemia & 61 & 71,8 & 30 & 69,8 & 31 & 73,8 & 0,679 \\
\hline Hipertensión Arterial & 41 & 48,2 & 23 & 53,5 & 18 & 42,9 & 0,327 \\
\hline Corticoterapia & 16 & 18,8 & 9 & 20,9 & 7 & 16,7 & 0,615 \\
\hline Diabetes melitus & 25 & 29,4 & 11 & 25,6 & 14 & 33,3 & 0,433 \\
\hline Insuficiencia Cardiaca Congestiva ** & 4 & 4,7 & 3 & 7,0 & 1 & 2,4 & 0,616 \\
\hline Tumor sólido ** & 9 & 10,6 & 4 & 9,3 & 5 & 11,9 & 0,738 \\
\hline Neoplasia hematológica ** & 5 & 5,9 & 3 & 7,0 & 2 & 4,8 & 1 \\
\hline Cirrosis Hepática ** & 8 & 9,4 & 4 & 9,3 & 4 & 9,5 & 1 \\
\hline Insuficiencia Renal Crónica & 32 & 37,7 & 16 & 37,2 & 16 & 38,1 & 0,933 \\
\hline Infección por VIH ** & 8 & 9,4 & 5 & 11,6 & 3 & 7,1 & 0,713 \\
\hline Neutropenia & 10 & 11,8 & 5 & 11,6 & 5 & 11,9 & 1 \\
\hline Desnutrición & 46 & 54,1 & 25 & 58,1 & 21 & 50,0 & 0,451 \\
\hline Uso previo de antibióticos & 51 & 60,0 & 27 & 62,8 & 24 & 57,1 & 0,595 \\
\hline \multicolumn{8}{|l|}{ Mortalidad } \\
\hline Fallecidos ** & 10 & 11,8 & 7 & 16,3 & 3 & 7,1 & 0,313 \\
\hline
\end{tabular}

* Se reportan mediana, intervalo intercuartil y valor $\mathrm{p}$ por prueba de Mann Whitney

** Se realizó prueba exacta de Fisher en lugar de Chi cuadrado

t Otras: Enterobacter spp, Salmonella spp, Citrobacter freunii, Serratia app y Proteus mirabilis

₹ Sólo realizado con $25 \operatorname{BLEE}(+)$ y $28 \operatorname{BLEE}(-)$ que pertenecían al grupo con infección nosocomial 
(UCI) fue de 13,9\%, estudios realizados en países como Brasil (4) dan cuenta de que el $80 \%$ de estos pacientes son manejados en $\mathrm{UCl}$, resultados que podría sugerir un cuidado no óptimo para nuestros pacientes portadores de bacteriemia por enterobacterias. Esta situación podría ser explicada por la sobrecarga de demanda de las unidades de cuidados intensivos, que generaría una «sub-admisión», situación que debería ser explorada en estudios futuros.

Entre las principales limitaciones, tenemos la naturaleza descriptiva del estudio, así como el tamaño de la muestra, y a pesar de que hallamos homogeneidad en las variables en estudio, un tamaño muestral mayor, permitiría hallar o descartar con mayor confianza la presencia de factores asociados que permitan un rápido reconocimiento, una terapia empírica apropiada, establecer si hay mayor mortalidad en los pacientes que desarrollan infecciones con enterobacterias productoras de BLEE y ratificar esta alarmante frecuencia de infecciones por este tipo de bacterias.

Los hallazgos de altas frecuencias de infecciones por patógenos productores de BLEE en la comunidad y en pacientes hospitalizados son recurrentes, por lo que se deberían tomar cultivos de manera imperativa en todos los casos, ante la posibilidad de estar frente a la presencia de gérmenes productores de BLEE, de manera que se pueda dirigir la terapia antibiótica de manera óptima.

En conclusión, la mitad de bacteriemias producidas por enterobacterias en pacientes hospitalizados de nuestro medio, serían producidas por gérmenes productores de BLEE, siendo $E$. coli y $K$. pneumoniae las especies más frecuentes, y de estas, el $40 \%$ son adquiridas en la comunidad.

Agradecimientos: A la Dra. Aida Palacios, jefa del departamento de Patología Clínica y Anatomía Patológica del Hospital Nacional Cayetano Heredia, por brindarnos las facilidades en la recolección de datos. Al Dr. Edward Michael Reyes Díaz, por la asesoría estadística.

Contribuciones de autoría: AFS, MNM, ABZ, GM participaron en la concepción y diseño del artículo, análisis e interpretación de datos. AFS, MNM, ABZ, CAG participaron en la recolección de resultados, CAG participó en el aporte de material de estudio, AFS, MNM, ABZ, CAG, GM participaron en la redacción del artículo; revisión crítica del artículo; aprobación de la versión

Conflictos de interés: Los autores declaran no tener conflictos de interés.

Fuentes de financiamiento: Autofinanciado.

\section{REFERENCIAS BIBLIOGRÁFICAS}

1. Adrianzén D, Arbizu A, Ortiz J, Samalvides F. Mortalidad por bacteriemia causada por Escherichia coli y Klebsiella spp. productoras de beta lactamasas de espectro extendido: cohorte retrospectiva en un hospital de Lima, Perú. Rev Peru Med Exp Salud Pública. 2013;30(1):18-25.

2. Tumbarello M, Sali M, Trecarichi EM, Leone F, Rossi M, Fiori B, et al. Bloodstream Infections Caused by Extended-Spectrum- $\beta$-Lactamase Producing Escherichia coli: Risk Factors for Inadequate Initial Antimicrobial Therapy. Antimicrob Agents Chemother. 2008 Sep;52(9):3244-52. doi: 10.1128/ AAC.00063-08.

3. Wang $S$, Zhao $S$, Xiao $S$, Gu F, Liu Q, Tang J, et al. Antimicrobial Resistance and Molecular Epidemiology of Escherichia coli Causing Bloodstream Infections in Three Hospitals in Shanghai, China. PLoS One. 2016;11(1):e0147740. doi: 10.1371/journal.pone.0147740.

4. Tuon FF, Kruger M, Terreri M, PenteadoFilho SR, Gortz L. Klebsiella ESBL bacteremia-mortality and risk factors. Braz J Infect Dis. 2011;15(6):594-8.

5. Echeverri-Toro LM, Rueda ZV, Maya W, Agudelo Y, Ospina S. Klebsiella pneumoniae multi-resistente, factores predisponentes y mortalidad asociada en un hospital universitario en Colombia. Rev Chil Infectol 2012;29(2):175-82.

6. Ramírez-Alfaro C, Villalobos-Vindas J. Análisis de las bacteremias por Klebsiella pneumoniae en pacientes del Hospital México. Acta Med Costarric. 2016;58(2):62-8.

7. Lee C, Su L, Chen F, Tang Y, Chien C, Liu J. Clinical and microbiologic characteristics of adult patients with recurrent bacteraemia caused by extended-spectrum- $\beta$-lactamase producing Escherichia coli or Klebsiella pneumoniae. Clin Microbiol Infect. 2015;21(12):1105.e1-8. doi: 10.1016/j.cmi.2015.07.025.

8. Sangare S, Maiga A, Guindo I, Maiga A, Camara N, Dicko O, et al. Prevalence of ESBL-producing Enterobacteriaceae isolated from blood cultures in Mali. J Infect Dev Ctries. 2016;10(10):10591064. doi: $10.3855 /$ jidc.7536.

9. Palma N, Gomes C, Riveros M, García W, Martínez-Puchol S, Ruiz-Roldán L, et al. Virulence factors profiles and ESBL production in Escherichia coli causing bacteremia in Peruvian children. Diagn
Microbiol Infect Dis. 2016;86(1):70-5 doi: 10.1016/j.diagmicrobio.2016.05.017.

10. Winokur PL, Canton R, Casellas JM, Legakis N. Variations in the Prevalence of Strains Expressing an Extended-Spectrum b-Lactamase Phenotype and Characterization of Isolates from Europe, the Americas, and the Western Pacific Region. Clin Infect Dis. 2001;32 Suppl 2:S94-103.

11. García A, García-Váquez E, Gómez J, Canteras M, Hernandez-Torres A, Ruiz J. Bacteriemia por Escherichia coli: factores predictivos de presencia de bacterias productoras de betalactamasas de espectro extendido e influencia de la resistencia en la mortalidad de los pacientes. Med Clin (Barc). 2011;136(2):56-60.

12. Delgado-Capel M, Gabillo A, Elias L, Yébenes J, Sauca G, Capdevilla J. Características de la bacteriemia relacionada con catéter venoso periférico en un hospital general. Rev Esp Quimioter 2012;25(2):129-33.

13. Martelius T, Jalava J, Kärki T, Möttönen T, Ollgren J, Lyytikäinen O. Nosocomial bloodstream infections caused by Escherichia coli and Klebsiella pneumoniae 
resistant to third-generation cephalosporins, Finland, 1999-2013: Trends, patient characteristics and mortality. Infect Dis (Lond). 2016;48(3):229-34. doi: $10.3109 / 23744235.2015 .1109135$.

14. Yang CC, Wu CH, Lee CT, Liu HT, Chen $\mathrm{JB}$, Chiu $\mathrm{CH}$, et al. Nosocomial extended-spectrum beta-lactamase- producing Klebsiella pneumoniae bacteremia in hemodialysis patients and the implications for antibiotic therapy. Int J Infect Dis. 2014;28:37. doi: 10.1016/j.ijid.2014.07.012..

15. Clinical and Laboratory Standards Institute (CLSI). Performance Standards for Antimicrobial Susceptibility Testing. 26th ed. CLSI supplement M100. Wayne, PA: Clinical and Laboratory Standards Institute; 2016.
16. García C. Resistencia antibiótica en el Perú y América Latina. Acta Med Per. 2012;29(2):99-103.

17. Jordá L, Vila A, Lanza A, Bonvehi P, Nazar J, Mikietuk A, et al. Utilidad del sistema VITEK en la identificación bacteriana y estudios de sensibilidad antimicrobiana. Acta Bioquím Clín Latinoam. 2005;39(1):19-25.

18. Maguiña-Vargas C; Ugarte-Gil C, Montiel M. Uso adecuado y racional de los antibióticos. Acta méd. peruana. 2006;23(1):15-20.

19. Castillo-Tokumori F, Irey-Salgado C, Málaga G. Worrisome high frequency of extended-spectrum beta-lactamase-producing Escherichia coli in community-acquired urinary tract infections: a case-control study. Int J Infect Dis. 2017;55:16-19. doi: 10.1016/j.ijid.2016.12.007.
20. Sánchez M, Claudio A, Kubiak B, Sosa A, Yrala G, Torrado U. Iniciativa de Enfermedades Infecciosas en América del Sur (SAIDI): Estudio sobre los factores determinantes del uso de antibióticos en consumidores de El Callao, Perú. 2006. Gaithersburg, MD: Links Media, APUA, DATUM, para la Agencia de los Estados Unidos para el Desarrollo Internacional (USAID).

Correspondencia: Germán Málaga

Dirección: Las cantutas 479-2, Casuarinas Sur. Santiago de surco

Correo electrónico: German.malaga@upch.pe

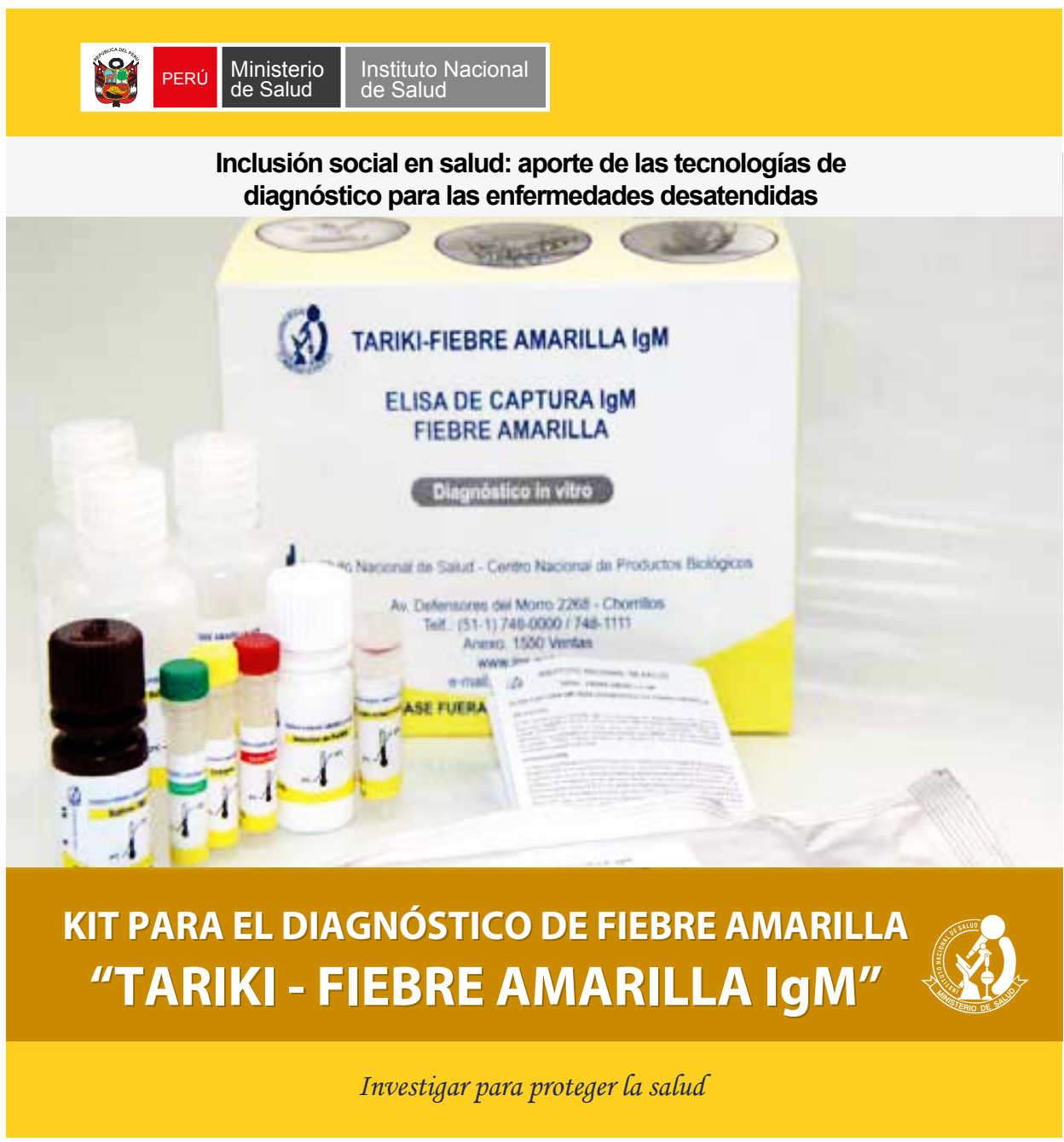

